\title{
Countywide survey of the current practice of chlamydia detection in primary care
} Julia Shefras, BChir, MRCOG, Specialist Registrar in Obstetrics and Gynaecology, St Michael's Hospital, Bristol, UK; Steve
Edmondson, MB BS, FRCPath, Consultant Microbiologist, Cheltenham General Hospital, Cheltenham, UK; Cliodna McNulty,
MB BS, FRCPath, Consultant Medical Microbiologist and PHLS Primary Care Co-ordinator, Public Health Laboratory Service (PHLS), Gloucester, UK

Correspondence: Julia Shefras, Department of Gynaecology, St Michael's Hospital, Bristol BS2 8EG, UK. Tel: +44 (0) 117 9285810

(Accepted 22 ${ }^{\text {nd }}$ May 2002)

The Journal of Family Planning and Reproductive Health Care 2002: 28(3): 145-148

\begin{abstract}
Background. The Chief Medical Officer's (CMO's) Advisory Group on Chlamydia trachomatis (chlamydia) published its report in 1998 and a national screening programme is anticipated. Meanwhile the Public Health Laboratory Service (PHLS) reports that the number of positive diagnoses of genital chlamydia continued to rise throughout the last decade.
\end{abstract}

Objectives. To consider the current practice of Gloucestershire general practitioners (GPs) for detecting genital chlamydia infections, and based on the findings to help the development of local guidelines and sexual health service provision.

Design. Questionnaire survey.

Setting. Primary care groups within a single English county.

Methods. A questionnaire survey was sent to GPs. Responses were handled anonymously and pooled for analysis.

Main outcome measures. Response rates from GPs on the types of tests used for detection of chlamydia infection, how frequently they test different patient groups for chlamydia infection and attitudes to contact tracing.

Results. In women, opportunistic screening is not routinely performed and the rate of diagnostic testing varies with presentation. The rate of testing is comparatively lower in men and over 50\% of GPs refer symptomatic men directly to a genitourinary medicine (GUM) clinic. Fewer than 50\% of respondents always or usually forward details to GUM for contact tracing.

Conclusions. Opportunistic screening is performed by a proportion of GPs but there is scope for more screening in higher risk groups. There is scope to increase the number of tests for chlamydia infection in patients presenting with symptoms. A programme of training and education in conjunction with guidelines may have merit in unifying practice and making detection and management of chlamydia more effective. This will have financial and resource implications.

\section{Key message points}

- Screening for chlamydia already occurs in primary care.

- Higher rates of screening in addition to testing in symptomatic attenders seem possible.

- There is a need for guidance on who should be tested and what tests should be used.

- The role of genitourinary medicine (GUM) clinics when chlamydia is detected in primary care needs to be clarified

\section{Introduction}

The Chief Medical Officer's (CMO's) Advisory Group on Chlamydia trachomatis published its report in $1998 .{ }^{1}$ It states that 'action is required to reduce the prevalence and morbidity associated with chlamydia infection'. While recognising that there is currently no national policy it did propose possible options with respect to future opportunistic and targeted screening, in addition to identifying high-risk groups in which opportunistic/targeted screening is reasonable. The report also described patient groups in which diagnostic testing is advisable.

Data from the Public Health Laboratory Service (PHLS) demonstrate that numbers with sexually transmitted diseases, and in particular chlamydia, are increasing. ${ }^{2}$ The best approach to screening is not yet clear, with questions such as the relative merits of targeted versus opportunistic screening and whether to screen men yet to be answered. The Chlamydia Screening Study (ClaSS) project, which started in February 2001, aims to address these issues. 3,4

Surveys of practice by general practitioners (GPs) in the UK, with respect to aspects of chlamydia detection and management, have been reported previously. One countybased study described development of evidence-based guidelines for GPs as a result of the survey. ${ }^{5}$ Another identified the need for guidelines for the management of chlamydia in the community in addition to adequate provision in GUM for the referrals. ${ }^{6}$

We decided to try and expand on the current knowledge regarding chlamydia testing in primary care and conducted a survey. The aims of this survey were to evaluate current practice in primary care in Gloucestershire with respect to chlamydia testing and from this make recommendations in order to optimise detection rates and management. It was also hoped that analysis of the responses (and free-text comments) would provide insight on issues that might impede implementation of a national screening policy.

\section{Methods}

A questionnaire was designed for GPs and primary care practice nurses (Table 1). Free-text comments were invited at the end of the survey. A draft questionnaire was piloted among a selection of health professionals including two GP registrars, two consultant gynaecologists, two public health/microbiology laboratory consultants and two nurse practitioners with specialist experience in the provision of sexual health. The final questionnaire was a result of feedback and opinion from these health professionals. The questionnaire was then sent to all GPs within the county. A 
total of 281 questionnaires reached their destination (postal failure meant that $10 / 19$ of one primary care group and only $1 / 13$ surgeries in another were sampled). The response rate to the successfully sent questionnaires was $61.2 \%$. In order to get an idea about the number of chlamydia tests requested by GPs, PHLS, Gloucester, UK and Microbiology Laboratory, Cheltenham, UK were asked to provide figures for the total number of tests and positive rates among men and women tested in primary care (Tables 2 and 3). These two laboratories serve the primary care groups surveyed.

\section{Results}

Table 1 depicts the questions and the GPs' responses.
Regarding methods of chlamydia testing

The results show that $93 \%$ of respondents always/usually do an endocervical swab, $15 \%$ always/usually do concurrent urethral swab, $7 \%$ always/usually test for chlamydial antibody titres and $56 \%$ occasionally do titres. Several respondents commented that they use antibody titers only in cases of suspected infertility. When testing for chlamydia, $90 \%$ of respondents always/usually do concurrent high vaginal swab (HVS) and 47\% always/usually do concurrent endocervical swabs for gonorrhoea.

Regarding chlamydia testing of female patients under 25 The results show that $53 \%$ of the GPs always/usually test

Table 1 Questions with general practitioner $(G P)$ responses expressed in absolute numbers and percentages (rounded up to the nearest whole number)

Questions

Absolute numbers

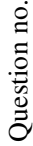

Percentage

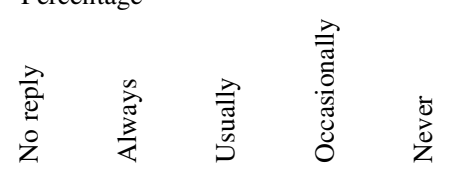

When investigating for chlamydia do you?

Swab endocervix

Swab urethra

Do chlamydia AB titres

Do other

Do concurrent HVS

Do concurrent endocervical swab for gonorrhoea

Which women do you test?

$<25$ with IMB

$<25$ with dyspareunia

$<25$ with pelvic pain

$<25$ with infertility

$\geq 25$ with IMB

$\geq 25$ with dyspareunia

$\geq 25$ with pelvic pain

$\geq 25$ with infertility

Which women do you screen ?

$<25$ attending for cervical smear

$\geq 25$ attending for cervical smear

$<25$ needing VE, e.g. for coil, FP, etc.

$\geq 25$ needing VE, e.g. for coil, FP, etc.

With respect to teenage boys and men

Do you see this group of patients?
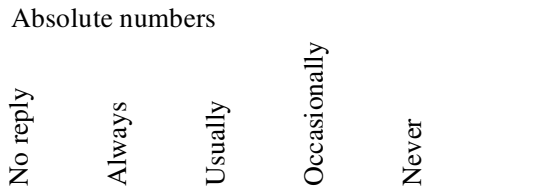

$\begin{array}{rrr}3 & 144 & 16 \\ 9 & 10 & 16 \\ 9 & 3 & 9 \\ 153 & 0 & 0 \\ 7 & 121 & 32 \\ 47 & 69 & 12\end{array}$

9
69
96
4
9
25

0
68

55

$\begin{array}{rrrrr}2 & 84 & 9 & 5 & 0 \\ 5 & 6 & 9 & 40 & 40 \\ 5 & 2 & 5 & 56 & 32 \\ 89 & 0 & 0 & 2 & 9 \\ 4 & 71 & 19 & 5 & 2 \\ 27 & 40 & 7 & 15 & 11\end{array}$

For men with unrelated Sxs do you screen opportunistically?

With EMU $<25$

With urethral swab $<25$

With EMU $\geq 25$

With urethral swab $\geq 25$

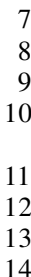

$\begin{array}{rrrr}8 & 8 & 8 & 63 \\ 7 & 3 & 5 & 67 \\ 5 & 19 & 25 & 60 \\ 5 & 16 & 19 & 66 \\ & & & \\ & \text { Yes } & \text { No } & \\ 3 & 149 & 20\end{array}$

\section{5}

90

63

66

For men with related Sxs do you screen?

With EMU $<25$

With urethral swab $<25$

Or refer directly to GUM $<25$

20
21
22
23

19
24
20
23

0
0
0
1

$\begin{array}{ll}2 & 19 \\ 2 & 10 \\ 3 & 14 \\ 1 & 10\end{array}$

$\begin{array}{ll}132 & 11 \\ 136 & 14 \\ 135 & 12 \\ 137 & 13\end{array}$

$\begin{array}{ll}9 & 26 \\ 4 & 4 \\ 4 & 49 \\ 4 & 4 \\ 8 & 19 \\ 4 & 29 \\ 2 & 35 \\ 5 & 3\end{array}$

29
19
12
21

35
26
23
28

With EMU $\geq 25$

With urethral swab $\geq 25$

Or refer directly to GUM $\geq 25$

$\begin{array}{rrrrrrrrrr}39 & 23 & 30 & 47 & 33 & 23 & 13 & 18 & 27 & 19 \\ 42 & 13 & 16 & 59 & 42 & 25 & 8 & 9 & 35 & 25 \\ 26 & 37 & 62 & 43 & 4 & 15 & 22 & 36 & 25 & 2 \\ & & & & & & & & & \\ 42 & 23 & 29 & 43 & 35 & 25 & 13 & 17 & 25 & 20 \\ 41 & 13 & 13 & 58 & 47 & 24 & 8 & 8 & 34 & 27 \\ 26 & 36 & 54 & 51 & 5 & 15 & 21 & 32 & 30 & 3\end{array}$

For contact tracing do you?

Forward details to GUM

Advise patient to inform partner

24
25
26
27
28
29

Are you designated GP/PN for particular institute? $\quad 3$

Do you have a practice screening policy?

$\begin{array}{rrrrr}30 & 26 & 45 & 37 & 32 \\ 31 & 9 & 96 & 55 & 10 \\ & & \text { Yes } & \text { No } & \\ 32 & 2 & 20 & 150 & 0 \\ 33 & & \text { Yes } & \text { No } & \\ & 3 & 10 & 159 & \end{array}$

32
2
0

$\begin{array}{rrrrr}15 & 26 & 22 & 19 & 19 \\ 5 & 56 & 32 & 6 & 1 \\ & \text { Yes } & \text { No } & & \\ 1 & 12 & 88 & 0 & 0 \\ & \text { Yes } & \text { No } & & \\ 2 & 6 & 93 & & \end{array}$

AB, Antibody; EMU, early morning urine; FP, family planning; GUM, genitourinary medicine; HVS, high vaginal swab; GP, general practitioner; IMB, intermenstrual bleeding; PN, practice nurse; VE, vaginal examination. 
Table 2 Chlamydia samples and numbers testing positive, taken by Gloucestershire primary care groups and sent to Public Health Laboratory Service (PHLS), Gloucester, UK (1 July 2000 to 30 June 2001)

\begin{tabular}{lllllll}
\hline & \multicolumn{3}{c}{ Female } & \multicolumn{5}{c}{ Male } \\
\hline Age of patient (years) & $\leq 25$ & $>25$ & Total & $\leq 25$ & $>25$ & Total \\
Tests (n) & 851 & 1816 & 2669 & 107 & 305 & 457 \\
Positive tests (n) & 89 & 36 & 125 & 17 & 54 & 71 \\
Positive (\%) & 10.5 & 2 & 4.7 & 15.9 & 15.4 & 15.5
\end{tabular}

Table 3 Chlamydia samples and numbers testing positive, taken by Gloucestershire primary care groups and sent to Cheltenham Microbiology Departments, Cheltenham, UK (1 July 2000 to 30 June 2001)

\begin{tabular}{llll}
\hline Gender & Female & & \\
\hline Age of patient (years) & $\leq 25$ & $>25 \leq 45$ & Total \\
Tests (n) & 1426 & 2539 & 3965 \\
Positive tests (n) & 122 & 50 & 172 \\
Positive (\%) & 8.6 & 2 & 4.3 \\
\hline
\end{tabular}

women presenting with intermenstrual bleeding, 77\% always/usually test those presenting with dyspareunia, $86 \%$ always/usually test women with pelvic pain, $71 \%$ always/usually test women with infertility, $10 \%$ always/usually test women presenting for a cervical smear and $26 \%$ screen women needing vaginal examination before coil fitting or other family planning advice. Similar results were seen in testing female patients over 25 years old for the same reasons as given above (see Table 1).

\section{Regarding male chlamydia testing}

A total of $12 \%$ of GPs do not see teenage boys or men; the majority of GPs (about 90\%) do not screen men opportunistically. When investigating men with urogenital symptoms, $48 \%$ of respondents always/usually test using either early morning urine (EMU) or urethral swabs and $56 \%$ of responders always/usually refer directly to GUM clinic.

\section{Regarding contact tracing}

A total of $48 \%$ of respondents always/usually forward details to GUM for such tracing. GPs also noted in free-text comments that patients are often unwilling to attend a GUM clinic.

Three practices had a written policy for some clinical situations (e.g. pre-coil fitting). The cut-off age for testing in women varied between practices, with some quoting 30 years old and others 25 years old as the upper limit in women.

\section{Discussion}

When testing patients for chlamydia infection $91 \%$ of GPs responded that they always/usually performed an endocervical swab for chlamydia. This finding compares favourably with a survey of GP practices in which one-third of GPs described using an inappropriate method of detection. ${ }^{6}$ Response rates to Question 1, however, do suggest possible confusion over best practice. Whereas comparable numbers of chlamydial endocervical swabs and HVS are always/usually performed together $(90 \%$ of the time), concurrent endocervical swabs for gonorrhoea are done only half as often (47\% always/usually). In women where sexually transmitted infections (STIs) are suspected it is reasonable to do concurrent endocervical swabs for microscopy and culture. In this way gonorrhoea in the endocervix may be detected. HVS is not indicated if an asymptomatic woman is simply being screened for chlamydia (except in pre-termination screening when it is sometimes policy to screen for asymptomatic bacterial vaginosis).

A notable finding from PHLS in Gloucestershire is that in women up to 25 years old from whom an HVS is taken, only $40 \%$ have a chlamydial swab (data from PHLS, Gloucestershire, UK). This practice represents missed opportunities to screen and/or test for chlamydia in a considerable number of women who are undergoing microbiological investigation of the lower genital tract anyway. Clear guidelines with respect to testing methods have the potential to increase the testing rate for chlamydia, reduce the number of unnecessary tests and identify what tests need to be done.

An important issue raised by several of the GPs (and practice nurses) was the possibility of using less invasive tests. Both laboratories acknowledge this issue and anticipate the possibility of more acceptable and easier testing methods in the future. At present female urine testing for chlamydia is not routinely performed in Gloucestershire.

\section{Testing in different patient groups}

Tables 2 and 3 show that of women who are currently tested in primary care in Gloucestershire, both symptomatic and asymptomatic, those aged under 25 years have the higher rate of lower genitourinary chlamydia. (It is not possible to comment on the relative contributions of diagnostic testing and screening to these figures.) The rate drops dramatically in the over-25 age group (8.6-10.5\% versus 2\%). Unfortunately the questionnaire only enquired about testing in women undergoing a pelvic examination. There was no enquiry about attendance for non-gynaecological problems (when no pelvic examination would be undertaken). National screening policy may require screening in this group.

\section{Symptomatic women}

Intermenstrual bleeding, dyspareunia, pelvic pain and infertility can all be caused by chlamydial infection. The result of the survey show that for women under $25,38 \%$ of responders occasionally/never test patients with intermenstrual bleeding, $20 \%$ of responders occasionally/ never test patients with dyspareunia, $12 \%$ occasionally test those with pelvic pain and $26 \%$ occasionally/never test women with infertility. The figures for GPs occasionally/never testing women over 25 with intermenstrual bleeding, dyspareunia, pelvic pain and infertility are $48 \%, 28 \%, 24 \%$ and $34 \%$, respectively. It appears that in primary care there is a lot of room for increased testing of women presenting with such complaints.

One of the weaknesses of the survey is that when testing for chlamydia in women with pelvic pain, infertility and dyspareunia the survey did not ask specifically what type of testing was undertaken. That would have helped to see whether swabs for gonorrhoea were taken. Testing for gonorrhoea would be indicated in such cases.

It is not clear why $7 \%$ of responders always/usually requested chlamydia antibody titres and some 56\% occasionally did so. Future local guidelines should mention that chlamydia antibody titres are not currently recommended for diagnosis of urogenital infection with this micro-organism. It is unknown whether the antibody tests requested by primary care are carried out as part of a diagnostic work-up requested by secondary referral centres or whether GPs are carrying them out independently. It is 
hoped that evaluation of chlamydia antibody testing in primary care will be the subject of a further study.

\section{Testing men}

It was a surprise finding that some $12 \%$ of responders did not see teenage boys or men. Chlamydia is a common cause of sterile pyuria in men. PHLS, Gloucester, UK and Microbiology Laboratory, Cheltenham, UK will routinely run enzyme immunoassays (EIA) on such a sample and further request an early morning/first void urine sample if this test is negative. Thus there is time and cost saving inherent in sending this sample initially.

Table 2 demonstrates several interesting points with respect to testing men for chlamydia in primary care. Far fewer men than women are tested which is likely to correspond to their lower attendance rate at GP surgeries. Lower numbers might also be explained by the fact that in the survey over $50 \%$ of GPs responded that they always/usually directly refer men with genitourinary symptoms to a GUM clinic. Of those tested in primary care, there is no age difference in detection rate: positive rate is almost equal in the under-25s and the over-25s. With the results of the survey suggesting that opportunistic screening of men does not routinely occur in primary care it can be inferred that the vast majority of those who are testing positive present with genitourinary symptoms. Yet it is known that $50 \%$ of infected men are asymptomatic and high rates of infection are seen up to the age of 34 years (in contrast to women in whom the rate declines sharply above 24 years). Hence there are comparable numbers of asymptomatic men in the community for whom the only method of identification currently is contact tracing following diagnosis in their sexual contacts.

Response to the survey implies that fewer than $50 \%$ of GPs always/usually forward details to GUM for contact tracing. Clarification therefore is needed on the effectiveness of current methods of partner notification/ treatment and the optimum level of involvement of GUM. This is particularly pertinent in the light of the report of the CMO's Expert Advisory Group and the draft National Strategy for Sexual Health and $\mathrm{HIV}^{7}$ which both identify GUM and specialist sexual health teams as best placed to offer contact tracing. Failure to identify and treat the asymptomatic pool of infection within the community will limit the effectiveness of any screening programme. However, there is question about confidentiality and we acknowledge that many GPs may offer referral but the patient declines.

Effective management of chlamydia in the community does not stand in isolation. Patient education in healthy sexual practices, appropriate use of contraception, awareness of other diseases, potential problems with fertility, issues over contact tracing and avoidance of unwanted pregnancy are all important. Free-text comments reflect GPs' concerns about the time and resources necessary to provide this service, as well as the ethical issues involved. Psychological morbidity associated with a positive diagnosis of chlamydia is well recognised. ${ }^{8}$ If current practice is to be optimised and/or a national screening programme introduced it is likely that this the burden of providing education, management and follow-up will increase significantly in primary care. Greater public awareness and demand for testing is also likely to add to this burden.

The division of responsibility and the interaction between primary care and GUM has to be clearly defined for effective management of positive cases and contact tracing.

The results of this survey suggest that there is a need for education for GPs and clear guidelines on who should be tested and what tests should be performed. (Several GP comments implied that guidelines would indeed be welcome.) There is a need for staff training in primary care to enable effective (and appropriate) sexual history taking, counselling, testing, and follow-up. Four GPs commented that practice nurses did most of the vaginal swabs. Training and support of practice nurses is an important aspect of any proposed programme. The responses from practice nurses have not been discussed here, however their free-text comments were consistent in a call for guidelines.

An increase in the numbers of tests in primary care and the numbers of patients seen in GUM clinics, if those tested positive are referred, will have obvious financial and cost implications for these services.

\section{Conclusions}

This survey demonstrates that there is awareness of genital chlamydia infection in the community and there is evidence that screening already occurs to some extent. There is potential for a greater proportion of both symptomatic and higher-risk asymptomatic women to be tested, albeit with cost, training and resource implications. There is even greater scope to increase testing of symptomatic men for chlamydia. Effective contact tracing is necessary, particularly to identify asymptomatic men.

This report has been used to inform the development of guidelines for the county. A study has been set up by PHLS, Gloucestershire, UK to investigate the change in laboratory investigation of chlamydia (and urinary tract infections) secondary to implementation of guidelines in conjunction with outreach workshops and laboratory use data feedback. Strengthening links between GUM and primary care is integral to the programme.

Through developing better practice it is hoped that the currently perceived problems inherent in primary care testing will be reduced and that unified policy across the county will also allow comparative analysis of rates of chlamydia detection between different groups and over time. It is recognised that national recommendations for screening will have an impact on countywide guidelines that have been developed.

\section{Acknowledgements}

The author wishes to thank the following individuals for their help and support: Mrs Mary Pillai and Dr Margaret Logan, and Dr Alan Herring of Bristol PHLS. The author is grateful to Wally Palmer of Gloucestershire PHLS for data relating to chlamydia detection rates in Gloucestershire.

Statements on funding and competing interests

Funding. None declared.

Competing interests. None declared.

References

Chief Medical Officer. Main report of the CMO's expert advisory group on Chlamydia trachomatis. London: Department of Health, 1998.

PHLS, DHSS \& PS and the Scottish ISD(D) 5 Collaborative Group. Trends in sexually transmitted infections in the United Kingdom 1990 to 1999. London: Public Health Laboratory Service (PHLS), 2000.

www.chlamydia.ac.uk

ClaSS Study Group. Evidence is not (yet) enough for evidence based policy for chlamydia screening [Letter]. BMJ 2001; 322: 364 .

Stokes T, Bhaduri S, Schober P, et al. GPs' management of genital chlamydia: a survey of reported practice. Fam Pract 1997; 14: 455-460.

Joshi U, Dixon W. General practitioners' views on the screening for genital Chlamydia trachomatis infection and partner notification. Int J STD AIDS 2000; 11: 588-591.

The National Strategy for Sexual Health and HIV - consultation document. London: Department of Health, July 2001.

Duncan B, Hart G, Scoular A, et al. Qualitative analysis of psychosocial impact of diagnosis of Chlamydia trachomatis: implications for screening. BMJ 2001;322: 195-199. 